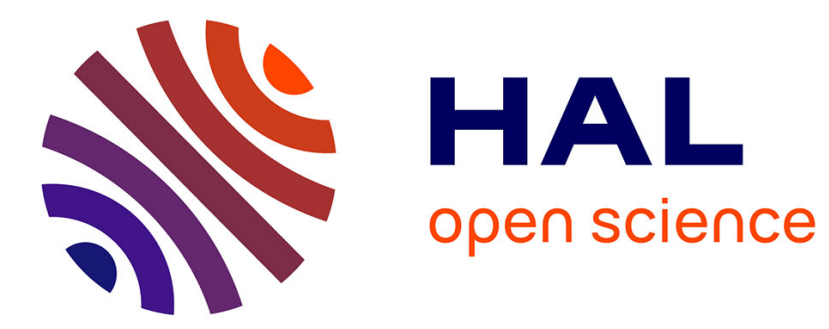

\title{
Numerical model for injection and transport in multilayers OLEDs
}

\author{
B. Masenelli, E. Tutis, M.N. Bussac, L. Zuppiroli
}

\section{To cite this version:}

B. Masenelli, E. Tutis, M.N. Bussac, L. Zuppiroli. Numerical model for injection and transport in multilayers OLEDs. Synthetic Metals, 2001, 122 (1), pp.141 - 144. 10.1016/S0379-6779(00)01350-3 . hal-01825332

\section{HAL Id: hal-01825332 \\ https://hal.science/hal-01825332}

Submitted on 17 Jan 2022

HAL is a multi-disciplinary open access archive for the deposit and dissemination of scientific research documents, whether they are published or not. The documents may come from teaching and research institutions in France or abroad, or from public or private research centers.
L'archive ouverte pluridisciplinaire HAL, est destinée au dépôt et à la diffusion de documents scientifiques de niveau recherche, publiés ou non, émanant des établissements d'enseignement et de recherche français ou étrangers, des laboratoires publics ou privés. 


\title{
Numerical model for injection and transport in multilayers OLEDs
}

\author{
B. Masenelli*1 ${ }^{1}$, E. Tutis ${ }^{1}$, M. N. Bussac ${ }^{2}$, L. Zuppiroli $^{1}$ \\ ${ }^{1}$ Laboratoire des solides semi-cristallins, EPFL, 1015 Lausanne, Switzerland \\ ${ }^{2}$ Laboratoire de physique théorique, Ecole Polytechnique, Palaiseau, France
}

\begin{abstract}
We present a recently developed numerical code for OLED simulation. This code contains a detailed description of contacts, charge transport and recombination. Its efficiency is briefly shown through examples of single-layer and bilayer devices and discussed in more detail through the investigation of the action of a LiF thin film on injection. In particular, we show how the code can help to discriminate between several possible explanations for this phenomenon.
\end{abstract}

Keywords: organic light-emitting diode, numerical model, transport, injection, recombination.

\section{Introduction}

Tremendous progress has been achieved concerning injection, transport and recombination of charge densities in OLEDs. Novel devices, using spacers, dipolar layers, new material multilayer stacks, have proved efficient in decreasing the voltage onset as well as increasing the external quantum efficiency. However, the reasons for this efficiency are not fully understood yet, and the behavior of typical single-layer and multilayer systems is not utterly clear. In order to clarify the fundamental mechanisms at stakes as well as to predict typical device behavior, a model is required. Several models, dealing with single layer or multilayer devices, have been proposed [1,2]. Despite their efficiency, they often describe injection in a restricted manner, which may reveal problematic to understand phenomena such as the role of insulating monolayers on injection.

\section{Model presentation}

In order to fully account for injection, transport and recombination in OLEDs, we have developed a numerical model. The model first takes advantage of the homogeneity of molecular organic layers. Hence, all significant quantities are calculated at each node of a one-dimensional (1D) array. The distance between two nodes is of physical relevance since it represents the actual distance between two molecules. It is therefore linked to the material density. At each node, energy levels and charge densities for both electrons and holes are to be calculated. The energy of the lowest unoccupied molecular orbital (LUMO) writes: 


$$
\tilde{\mathbf{E}}(x)=\mathbf{E}_{\mathbf{0}}+\mathbf{E}_{\mathbf{I F}}+\sum_{x_{m}} \mathbf{E}\left(x_{m}, x\right)
$$

where $\mathrm{E}_{0}$ is the bare LUMO energy, $\mathrm{E}_{\mathrm{IF}}$ is the image force contribution due to the presence of metal electrodes, and $\mathrm{E}\left(\mathrm{x}_{\mathrm{m}}, \mathrm{x}\right)$ is the contribution of charge at node $\mathrm{x}_{\mathrm{m}}$. A similar equation can be written for highest occupied molecular orbital (HOMO) levels. It is noteworthy that discreteness effects due to charge localization on molecules are taken into account, especially where they are most important, namely at the nodes near the electrodes (the description of charge localization effects is beyond the scope of this paper and will be published elsewhere [3]). Concerning the charge density evolution, three contributions are to be distinguished (cf. fig.1): the variation due to injection from electrodes, that due to transport within the organic layers, and the last one due to recombination. Focusing on electrons, one can write:

$$
\frac{\mathrm{d} n}{\mathrm{dt}}=\left(\frac{\mathrm{d} n}{\mathrm{dt}}\right)_{\text {injection }}+\left(\frac{\mathrm{d} n}{\mathrm{dt}}\right)_{\text {transport }}+\left(\frac{\mathrm{d} n}{\mathrm{dt}}\right)_{\text {recombination }}
$$

In our framework, injection is treated as a tunnelling current between electrode and several nodes. This accounts for long range tunneling. Transport is modelled as hopping between nearest-neighbor sites in local thermodynamic equilibrium. The hopping frequency is assumed to be constant (at typical electric field) and deduced from the macroscopic mobility value. Recombination is treated in the framework of Langevin theory, as being the result of a competition between the separating drift force and the attractive Coulomb force. As one can see, charge densities and energy levels are coupled in a non-explicit way. Thus, equations (1) and (2) must be solved self-consistently. Our code fulfills this criterion using a time-dependent procedure.

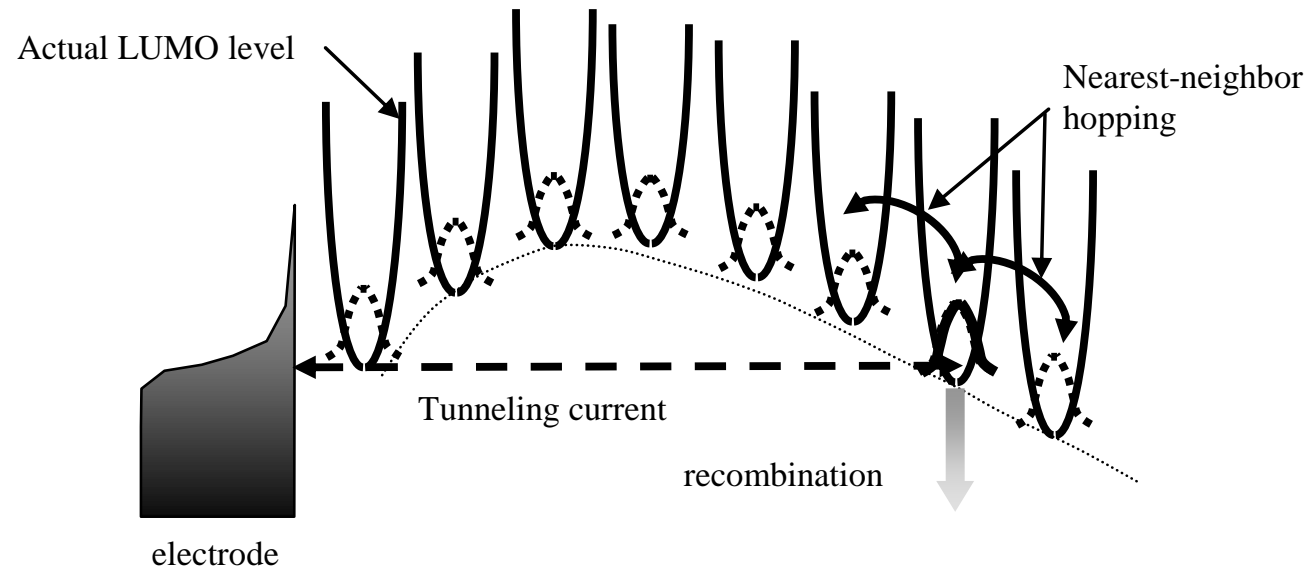

Figure 1: energy scheme representing the three mechanisms contributing to charge density variations: long range tunnel injection, nearest-neighbor hopping and recombination

\section{Results}

\subsection{Simulation of single-layer and bilayer devices}

First of all, the model is found to simulate single-layer devices accurately. Among others, we simulated some $\mathrm{Ca} / \mathrm{Alq}_{3} / \mathrm{Ca}$ devices fabricated in our laboratory [3] as well as devices made by the Los Alamos group [4]. For these simulations, only a small number of parameters (tunneling integral, position of the first node) had to be determined. This was made possible because available experimental data were numerous enough to provide a strong constraint on these parameters. Our 
model also provided an explanation of the pinning of the energy barrier at the metal/organic interface observed by Campbell et al. [5].

In a second step, we used the code to investigate bilayer devices. We thus fabricated several bilayer devices made of an aluminum cathode and a $\mathrm{O}_{2}$ plasma-treated ITO anode, surrounding an electron transport layer (ETL) of Zn(q-2-COOEt) $)_{2}$ and an emitting and hole transport layer (HTL) of a CN derivative of bicarbazyl [6]. The thickness of the ETL was varied from $15 \mathrm{~nm}$ to $60 \mathrm{~nm}$ while keeping the HTL thickness constant. This provided a set of experimental I-V and luminance-V curves that enabled the determination of the few unknown parameters. The code could simulate the experimental data successfully and revealed space charge effects due to hole accumulation in the ETL because of their low mobility in this material [3].

\subsection{Simulation of the action of a LiF thin film on electron injection.}

The simulation of both single-layer and bilayer devices revealed the dramatic influence of the injection interface on the device behaviour. We thus focused on the description on such interfaces, and particularly, on optimized injection interfaces. It is known that adding a thin film of insulating material such as $\mathrm{LiF}, \mathrm{MgO}$ or self-assembled monolayer can enhance electron injection. Among these materials, the most used is LiF. We thus concentrated on determining its action. Two mechanisms have been proposed to explain its favorable effect on injection. The first one relies on UPS measurements. These measurements showed that adding a small LiF layer on a metal (most of the time $\mathrm{Al}$ ) decreases its work function [7,8,9]. Reported values for this reduction span from $0.5 \mathrm{eV}$ to $1.5 \mathrm{eV}$ depending on the $\mathrm{LiF}$ thickness and on the group. The proposed explanation is the creation of a dipole within the LiF layer. This dipole would lower the metal work function. In the second hypothesis, the insulating film reduces the undesirable effect of the image force. Indeed, the image force induces the existence of a zone where the local electric field is opposed to the external one. This leads to a local current which may flow back to the electrode. By moving the first organic molecule away from the metal surface thanks to the LiF film, the width of this zone as well as the amplitude of the negative local field is reduced. Consequently, the total current coming from the electrode is enhanced.

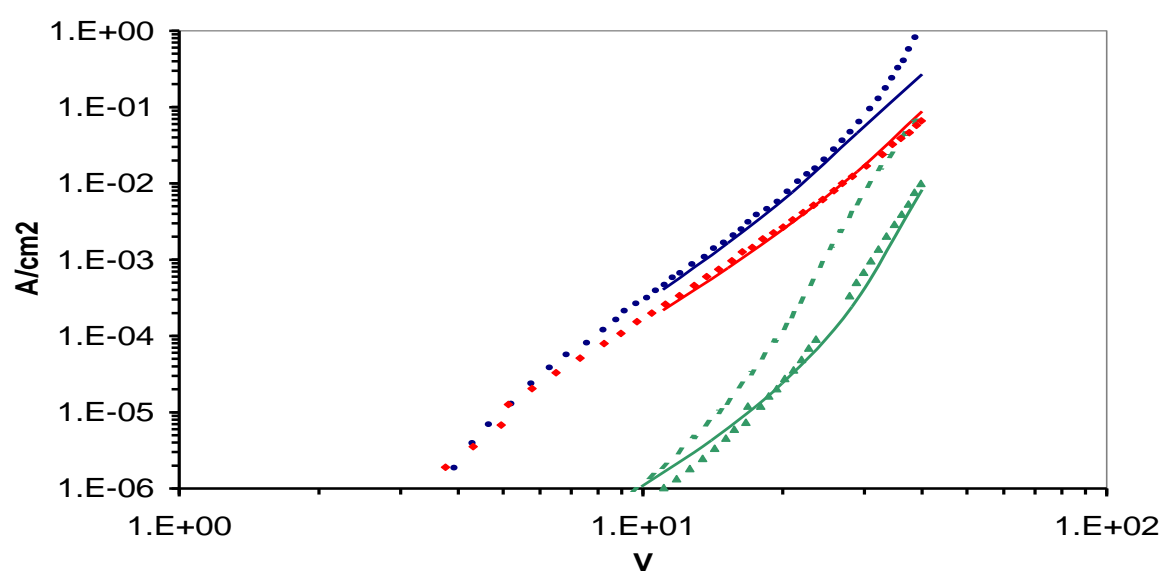

Figure 2: simulation of the effect of LiF on injection

We used our code to investigate these mechanisms. A recent paper by Stößel et al. [10] provided us with neat experimental data. They fabricated $\mathrm{Al} / \mathrm{LiF} / \mathrm{Alq}_{3}(300 \mathrm{~nm}) / \mathrm{Mg}$ devices with different thickness of LiF layer. We used these data for simulation. Fig. 2 reproduces the simulation results along with the experimental data. The triangle symbol curve is the experimental I-V curve for a device with no LiF. The full line curve on top of it is the result of the simulation for this device. The dotted curve is obtained from the previous one by moving the first molecule away from the electrode 
by $1 \mathrm{~nm}$. One can see that it does enhance the injection. But the enhancement is not large enough to reproduce the experimental one obtained with the addition of $0.2 \mathrm{~nm}$ (circle symbol) and $0.5 \mathrm{~nm}$ (square symbol) of LiF. Hence, the reduction of the backflow current alone cannot explain the action of LiF. We thus investigated the possibility of a dipole effect. We deposited 55nm thick Al layers capped with $0,0.5,0.6,1.5$ and $3 \mathrm{~nm}$ thick LiF layers. After deposition, the samples were moved from the deposition chamber to a nearby glove box for Kelvin probe measurements. The work function of $\mathrm{Al}$ capped layers versus $\mathrm{LiF}$ thickness is reproduced in fig.3. It is clear that by adding a $\mathrm{LiF}$ layer, the work function of $\mathrm{Al}$ is lowered typically by $0.35 \mathrm{eV}$ for a $6 \AA$ thick Lif layer, $0.5 \mathrm{eV}$ for a $30 \AA$ thick LiF layer.

Figure 3: decrease of the $\mathrm{Al}$ work function versus LiF thickness (Kelvin probe measurements)

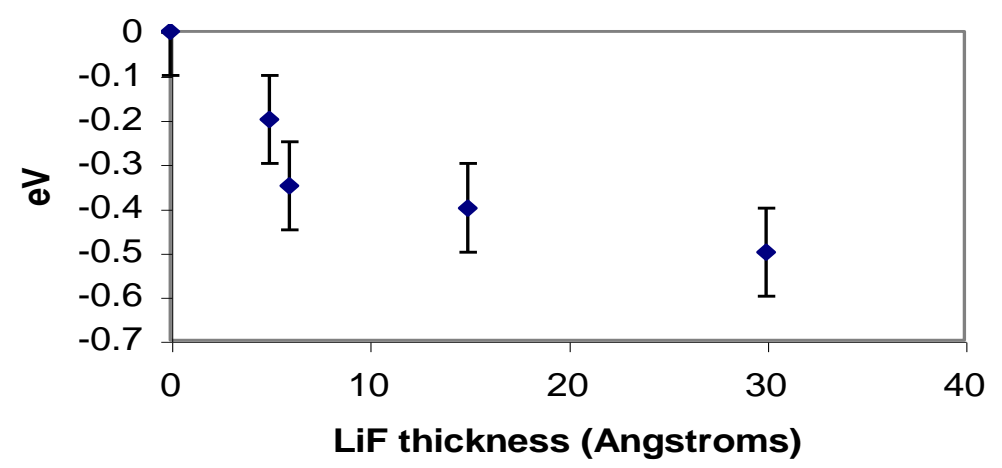

Consequently, we reduced the energy barrier between $(\mathrm{Al} / \mathrm{LiF})$ and $\mathrm{Alq}_{3}$ in the simulation, which was initially of $0.65 \mathrm{eV}$. The simulation of experimental devices with $2 \AA$ and $5 \AA$ thick LiF layers set the barrier height to $0.25 \mathrm{eV}$ (which is in fair accordance with both Kelvin probe measurements and UPS values). The only difference between these two simulations was the tunneling integral value (i.e. the value of the overlapping wave functions of the electron in the metal and on the first molecular site). The tunneling integral value was decreased by a factor 100 for the device having a 5 $\AA$ thick LiF layer. This accounts for the insulating action of LiF. The good agreement between the simulations and the experimental data shows that the dipole effect is necessary to explain the enhancement of injection induced by the presence of LiF. However, this dipole effect cannot always be invoked. In particular, for self-assembled organic monolayers which exhibit no dipole or even a dipole opposed to injection [11]. This type of device still needs to be investigated and modelled to fully account for the injection enhancement.

\section{References}

1 B. K. Crone, P. S. Davids, i. H. Campbell, D. L .Smith, J. Appl. Phys. 87, (2000), 1974

2 J. Staudigel, M. Stößel, F. Steuber, J. Simmerer, J. Appl. Phys. 86, (1999), 3895

3 E. Tutis, M. N. Bussac, B. Masenelli, M. Carrard, L. Zuppiroli, J. Appl. Phys. (submitted)

4 I. H. Campbell,, D. L. Smith, Appl. Phys. Lett., 85, (1999), 608

5 E. Tutis, M. N. Bussac, L. Zuppiroli, Appl. Phys. Lett., 81, (1999), 3880

6 N. Donzé, P. Péchy, M. Grätzel, M. Schaer, L. Zuppiroli, Chemp. Phys. Lett., 315, (1999), 405

7 R. Schlaf, B. A. Parkinson, P. A. Lee, K. W. Nebesny, G. Jabbour, B. Kippelen, N. Peyghambarian, N. R.

Armstrong, J. Appl. Phys, 84, (1999), 6729

8 H. Ishii, K. Sugiyama, E. Ito, K. Seki, Adv. Mat. 11, 8 (1999) 605

9 T. Mori, M. Suzuki, Y. Taga, SPIE conf. Proceedings, Denver, Colorado, July 1999, 367

10 M. Stößel, J. Staudigel, F. Steuber, J. Blässing, J. Simmerer, Appl. Phys. Lett., 76, (2000), 115

11 L. Zuppiroli, L. Si-Ahmed, K. Kamaras, F. Nüesch, M. N. Bussac, D. Ades, A. Siove, E. Moons, M. Grätzel, Eur. Phys. J. B 11, (1999), 505 